BNL-NT-07/7

\title{
Transition temperature in QCD with physical light and strange quark masses
}

\author{
Frithjof Karsch (for the RBC-Bielefeld collaboration) \\ Physics Department, Brookhaven National Laboratory, Upton, NY 11973, USA \\ E-mail: karsch@bnl.gov
}

\begin{abstract}
We present results from a calculation of the transition temperature in QCD with two light (up, down) and one heavier (strange) quark mass as well as for QCD with three degenerate quark masses. Furthermore, we discuss first results from an ongoing calculation of the QCD equation of state with almost realistic light and strange quark masses.
\end{abstract}

\section{Introduction}

Lattice calculations provide a unique first principle approach to the thermodynamics of QCD. They have the potential to produce definitive answers for basic bulk thermodynamic properties as well as the phase diagram of QCD. To reach this ambitious goal one has to control lattice discretization errors that lead to systematic deviations of lattice calculations from continuum physics, as well as finite volume effects that that can obscure the thermodynamic limit.

We present here results from a detailed analysis of the transition temperature in QCD with an almost physical value of the strange quark mass and several values of the light quark mass. This allows to perform an extrapolation to the chiral limit as well as to the physical point. Calculations have been performed for two different values of the lattice cut-off which allows to estimate the lattice discretization errors and perform an extrapolation to the continuum limit. The flavor dependence of these results are examined through an analogous analysis of QCD with three degenerate quark flavors. We also report on results from an ongoing study of the QCD equation of state with almost physical light and strange quark masses. All these calculations have been performed with an $\mathcal{O}\left(a^{2}\right)$ tree-level improved gauge action and an improved staggered fermion action (p4fat3) that also improves bulk thermodynamic observables at $\mathcal{O}\left(a^{2}\right)$. For further details on the calculational set-up we refer to [1, 2].

\section{The transition temperature}

In order to determine the transition temperature and its dependence on the light quark mass we have performed calculations with a fixed value of the bare strange quark mass 

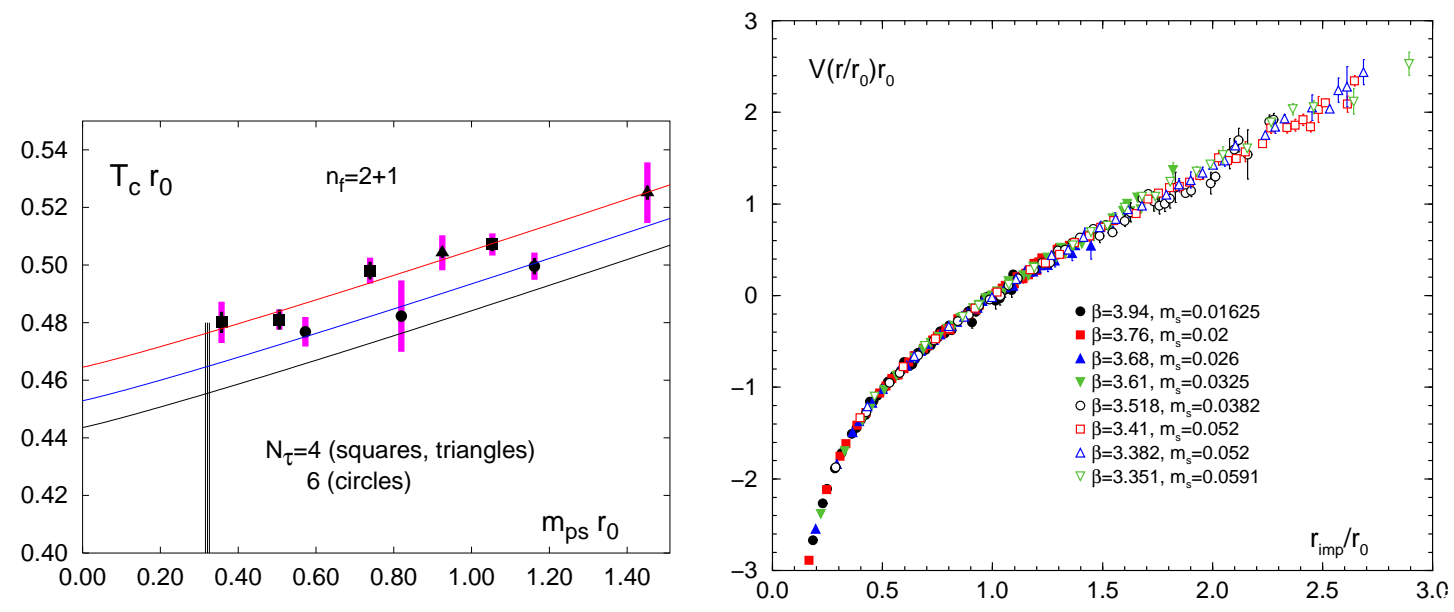

Figure 1. The transition temperature (left) calculated on lattices with temporal extent $N_{\tau}=4,6$ and the static quark potential (right) in units of $r_{0}$ calculated on $16^{3} \times 32$ lattices. $V(r)$ has been calculated for a wide range of gauge coupling $\beta$ in which the lattice cut-off varies by a factor 4 . This range also covers the the relevant parameter range of the finite temperature calculations.

and several (degenerate) values for the two light quark masses that correspond to light pseudo-scalar masses $\left(m_{p s}\right)$ ranging from $m_{p s} \simeq 150 \mathrm{MeV}$ to $m_{p s} \simeq 500 \mathrm{MeV}$. For non-zero values of the quark masses the transition to the high temperature phase of QCD is signaled by peaks in response functions. We have analyzed Polyakov loop and chiral susceptibilities to locate the transition point for the different quark mass values on lattices with temporal extent $N_{\tau}=4$ and 6 [1]. The spatial extent of the lattice has been varied between $N_{\sigma} / N_{\tau}=2$ and 4 . We generally observe only a weak volume dependence of the transition point and also find that the above susceptibilities as well as quark number susceptibilities in the light and strange quark sector [3] yield, within statistical errors, consistent results for the transition point.

In order to convert the results for the transition temperature to physical values one needs to set the scale through the calculation of zero temperature observables. Unlike hadronic observables in the light quark sector, which require a careful handling of chiral extrapolations, are known to be difficult to determine and in some cases are influenced by large discretization errors, the parameters of the static quark potential have been found to show only a mild dependence of the lattice cut-off [4]. We thus have performed extensive studies of the static quark potential, $V(r)$, on zero temperature lattices and extracted the scale parameter $r_{0}$ defined through the slope of $V(r)$, i.e. $\left(r^{2} \mathrm{~d} V(r) / \mathrm{d} r\right)_{r=r_{0}}=1.65$. Using the results obtained for $r_{0}$ in lattice units at the crossover couplings, which have been determined in finite temperature simulations on lattices with temporal extent $N_{\tau}=4$ and 6 , the transition temperatures can then be calculated as $T_{c} r_{0} \equiv r_{0} / N_{\tau} a$. Results for $T_{c} r_{0}$ and the static quark potential in units of $r_{0}$ are shown in Fig. 1. Results for $T_{c} r_{0}$ have then been extrapolated to the continuum limit using an ansatz that incorporates the quark mass dependence of $T_{c}$ through the 

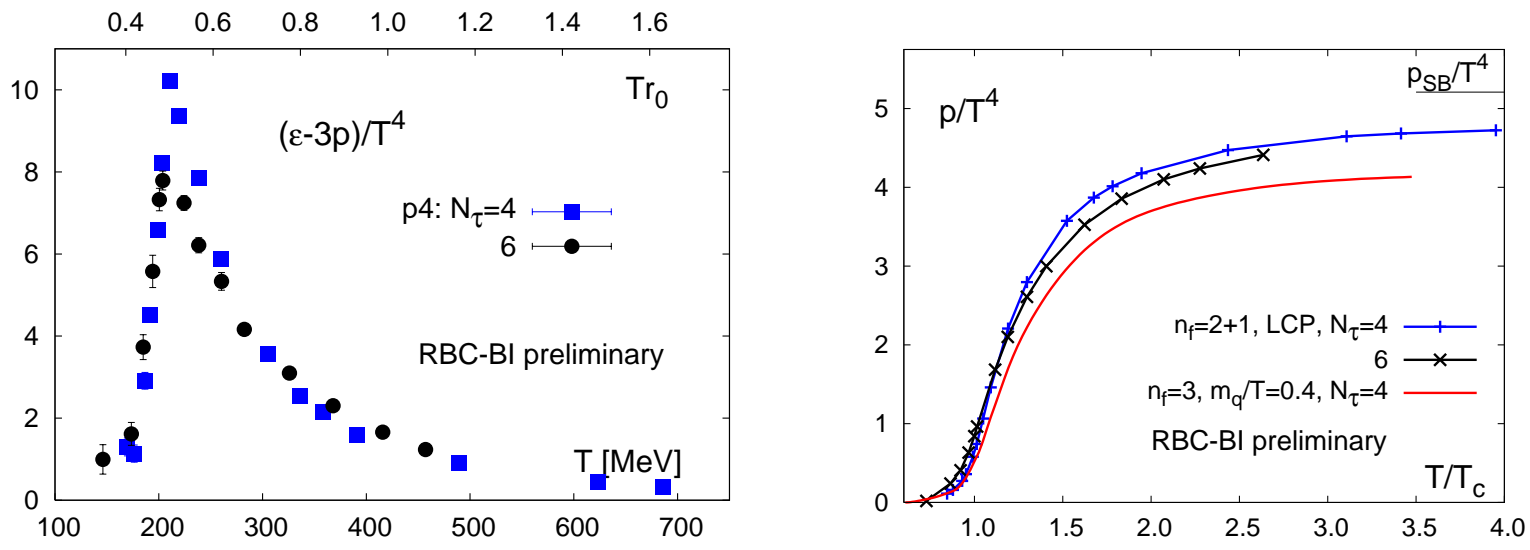

Figure 2. The trace anomaly, $(\epsilon-3 p) / T^{4}$, calculated in $(2+1)$-flavor QCD on lattices with temporal extent $N_{\tau}=4$ and 6 , respectively. Fig.2(right) shows the resulting pressure from Eq. 3 as well as earlier results obtained for 3-flavor QCD (see text).

calculated values of the lightest pseudo-scalar mass as well as a quadratic dependence on the lattice cut-off,

$$
\left(T_{c} r_{0}\right)_{\hat{m}_{l}, \hat{m}_{s}, N_{\tau}}=\left(T_{c} r_{0}\right)_{0, m_{s}, \infty}+A\left(m_{p s} r_{0}\right)^{d}+B / N_{\tau}^{2} .
$$

Here $d$ has been varied between $d=1$ and 2 to allow for uncertainties in the extrapolation to the chiral limit [1]. The continuum extrapolation is shown as the lower curve in Fig. 1(left). For the transition temperature at the physical point we find,

$$
T_{c} r_{0}=0.457(7)_{-2}^{+8} \Leftrightarrow T_{c}=192(7)(4) \mathrm{MeV}
$$

where we have used $r_{0}=0.469(7) \mathrm{fm}[5]$ to set the scale for $T_{c}$.

A similar analysis has been performed for QCD with three degenerate quark masses [2]. In this case the transition becomes first order for small but non-zero values of the quark mass. We find for the transition temperature in the chiral limit $T_{c} r_{0}=0.419(9)$ while in the same limit the transition temperature in $(2+1)$-flavor QCD is $T_{c} r_{0}=0.444(6)(12)$. We note that $T_{c} r_{0}$ in 3-flavor QCD is about $5 \%$ smaller than in $(2+1)$-flavor QCD. Moreover, the difference between the transition temperature in the chiral limit and at the physical point is only about $3 \%$. This is a direct consequence of the weak quark mass dependence of the transition temperature, which has been observed earlier [6]. A similarly weak quark mass dependence has also been observed in studies performed with another version of an improved staggered fermion action (asqtad) on lattices with temporal extent $N_{\tau}=4,6$ and 8 [7]. This analysis, however, lead to a $10 \%$ smaller transition temperature, $T_{c} r_{0}=0.402(29)$ than the value reported here. To resolve the origin of this discrepancy needs further investigations.

\section{The QCD equation of state}

We currently study the equation of state in (2+1)-flavor QCD [8] on lattices with temporal extent $N_{\tau}=4$ and 6 using the same calculational set-up used for the study of 
the transition temperature. Calculations are performed along a line of constant physics characterized by a fixed strange pseudo-scalar mass $m_{\bar{s} s} \simeq 660 \mathrm{MeV}$ and a fixed ratio of $m_{\bar{s} s}$ and the kaon mass, $m_{\bar{s} s} / m_{K} \simeq 1.3$. The light pseudo-scalar (pion) mass on this trajectory is about $220 \mathrm{MeV}$.

In Fig. 2 we show results for the trace anomaly, $\Theta_{\mu}^{\mu}(T) \equiv \epsilon-3 p$ in units of $T^{4}$ and the pressure $(p)$ which is deduced from $\Theta_{\mu}^{\mu}(T)$ using standard thermodynamic relations,

$$
\frac{p(T)}{T^{4}}-\frac{p\left(T_{0}\right)}{T_{0}^{4}}=\int_{T_{0}}^{T} \mathrm{~d} T^{\prime} \frac{1}{T^{\prime 5}} \Theta_{\mu}^{\mu}\left(T^{\prime}\right)
$$

We also included in Fig. 2(right) results for the pressure obtained earlier [9] for 3-flavor QCD with larger quark masses that have been held fixed in units of $T$. For these earlier calculations the light pseudo-scalar mass was about $770 \mathrm{MeV}$ in the transition region and would have been about $2 \mathrm{GeV}$ in the region around $T \simeq 3 T_{c}$ !

The results presented here for $(\epsilon-3 p) / T^{4}$ and $p / T^{4}$ in $(2+1)$-flavor QCD are entirely consistent with results obtained with the asqtad action [10]. In particular, results for $p / T^{4}$ obtained with the p4fat3 and asqtad actions, which both have a $\mathcal{O}\left(a^{2}\right)$ improved ideal gas limit, show little cut-off dependence in the entire temperature regime analyzed. This is in contrast to calculations performed with a variant of standard staggered fermions [11] which unlike p4fat3 and asqtad actions lead to large cut-off distortions of the high- $\mathrm{T}$ limit on lattices with temporal extent $N_{\tau}=4$ and 6 . We also note that we find no evidence for a significant broadening of the transition region when going closer to the continuum limit. Whether this happens for larger $N_{\tau}$, as a recent analysis of the transition temperature on lattices with temporal extent up to $N_{\tau}=10$ may suggest [12], has to be examined in more detail.

\section{Acknowledgments}

This manuscript has been authored under contract number DE-AC02-98CH1-886 with the U.S. Department of Energy.

\section{References}

[1] M. Cheng et al. (RBC-Bielefeld collaboration), Phys. Rev. D 74, 054507 (2006)

[2] M. Cheng et al. (RBC-Bielefeld collaboration), hep-lat/0612001, to be published in Phys. Rev. D

[3] Ch. Schmidt, hep-lat/0701019.

[4] C. Aubin et al., Phys. Rev. D70 (2004) 094505.

[5] A. Gray et al., Phys. Rev. D72 (2005) 094507.

[6] F. Karsch, E. Laermann, A. Peikert, Nucl. Phys. B 605 (2001) 579.

[7] C. Bernard et al., Phys. Rev. D 71, 034504 (2005).

[8] M. Cheng et al. (RBC-Bielefeld collaboration), in preparation

[9] F. Karsch, E. Laermann and A. Peikert, Phys. Lett. B 478, 447 (2000)

[10] C. Bernard et al., hep-lat/0611031

[11] Y. Aoki, Z. Fodor, S. D. Katz and K. K. Szabo, JHEP 0601, 089 (2006).

[12] Y. Aoki, Z. Fodor, S. D. Katz and K. K. Szabo, Phys. Lett. B 643, 46 (2006) 